\title{
Evolution of angiotensin II-mediated atherosclerosis in ApoE KO mice
}

\author{
JOHN CHA, VADIM IVANOV, SVETLANA IVANOVA, TATIANA KALINOVSKY, \\ MATTHIAS RATH and ALEKSANDRA NIEDZWIECKI
}

Dr Rath Research Institute, 1260 Memorex Drive, Santa Clara, CA 95050, USA

Received April 14, 2010; Accepted May 11, 2010

DOI: $10.3892 / \mathrm{mmr} 00000298$

\begin{abstract}
The dysregulation of renin-angiotensin systemsignaling is a contributing factor to the development of atherosclerosis and cardiovascular disease. We studied the progression of angiotensin II (AngII)-induced arterial wall atherosclerosis after its induction. Atherosclerosis was accelerated in ApoE (-/-) C57B6 mice by 4 weeks of continuous osmotic pump administration of AngII ( $23 \mathrm{nmol} / 24 \mathrm{~h}$ ). Changes in aortae were evaluated in these mice at 4 and 16 weeks after pump installation, as well as in control mice that were not administered AngII. In comparison to the non-AngII controls, AngII supplementation induced additional lesions in the aortic arch and its vessels 4 weeks after pump installation, indicating that AngII had an effect. These observations were grossly visible and were confirmed through histological analysis. Additionally, the AngII-mediated injuries continued to accelerate great vessel atherosclerosis after the discontinuation of AngII administration at 4 weeks. Atherosclerosis in the ApoE KO mice at 16 weeks post-AngII administration was increased by over 3-fold compared to the difference between lesion development in the ApoE KO mice at 4 weeks post-AngII and the age-matched control ApoE KO mice. While lesion size 12 weeks after cessation of AngII (16 week post-AngII group) increased $249 \%$ compared to the 4 week post-AngII group, differences between the rate of lesion formation in the 4 week post-AngII and non-AngII groups indicated a $70 \%$ increase and a linear relationship with time. Aortic dissections were present at 4 weeks post-AngII pump installation at high frequency compared to the unsupplemented controls, in which they were absent. Unexpectedly, aortic dissections were absent at 16 weeks post-AngII, indicating the presence of a healing process. The study suggests that excessive AngII initiates a cascade of pathological biochemical
\end{abstract}

Correspondence to: Dr Aleksandra Niedzwiecki, 1260 Memorex Drive, Santa Clara, CA 95050, USA

Email: author@drrath.com

Key words: atherosclerosis, angiotensin II, ApoE KO mice, aortic dissection events and plaque evolution that does not cease even after AngII administration is discontinued.

\section{Introduction}

Considerable progress has been made in characterizing the pathogenesis of atherosclerosis from its initiation to the development of end stage plaque rupture, thrombosis and arterial obstruction $(1,2)$. Understanding of its pathogenesis has been increased by observations made at every stage of the disease in studies with differing analytical perspectives and objectives. Despite continual refinement and innovation, tools for the study of the disease remain limited by numerous and considerable scientific, logistical and anthropological constraints.

Rodent models of atherosclerosis offer the advantages of higher data throughput and a shorter life cycle, thereby increasing efficiency and the speed of progress. Worldwide, cardiovascular medicine employs a few specific genetically engineered mouse models in their attempt to better understand human atherosclerosis $(3,4)$. Current state-of-the-art atherosclerosis studies uniformly use genetically modified hyperlipidemic, low density lipoprotein receptor (LDLR -/-) or apolipoprotein E (ApoE) (-/-) knockout (KO) mice, or crosses of these, to generate data regarding experimentally induced atherosclerosis (5).

Angiotensin II (AngII), a powerful vasoconstrictor, is believed to contribute to the development of hypertension through distorted signaling (6). Besides its role in vasoconstriction, AngII has been implicated in the acceleration of other pathological processes leading to the development of atherosclerosis and vascular disease, such as inflammation due to the induction of cytokines and matrix remodeling through the modulation of collagen synthesis and matrix metalloproteinase (MMP) expression (6). Modern clinical management of hypertension is almost entirely based on blocking various steps of the AngII-dependent signaling cascade. It has yet to be determined whether blockage of AngII signaling followed by the control of blood pressure allows for the healing of damaged vasculature.

In order to address this point, we used an animal model of atherosclerosis induced by continuous administration of AngII in ApoE KO mice with a genetically compromised lipid metabolism. The goal of this study was to evaluate the progression of atherosclerotic damage after the cessation of AngII supplementation. 
Table I - Treatment Summary

\begin{tabular}{lcccc}
\hline $\begin{array}{l}\text { Murine } \\
\text { groups }\end{array}$ & $\begin{array}{c}\text { Murine } \\
\text { genotype }\end{array}$ & $\begin{array}{c}\text { AngII treatment } \\
\text { (duration/age at implantation) }\end{array}$ & $\begin{array}{c}\text { Age at time of harvest } \\
\text { post-AngII treatment }\end{array}$ & $\begin{array}{c}\text { No. of weeks } \\
\text { after initiation }\end{array}$ \\
\hline $1(\mathrm{n}=9)$ & Wild Type & None & 36 weeks & N/A \\
$2(\mathrm{n}=9)$ & ApoE KO & None & 36 weeks & N/A \\
$3(\mathrm{n}=9)$ & ApoE KO & 4 weeks/32 weeks & 36 weeks & 4 weeks \\
$4(\mathrm{n}=9)$ & ApoE KO & 4 weeks/36 weeks & 52 weeks & 16 weeks \\
\hline
\end{tabular}

AngII treatment by continuous osmotic pump administration. N/A, not applicable.

\section{Materials and methods}

Animals. C57B6 ApoE (-/-) male mice $(\mathrm{n}=36)$ and wild-type male mice $(\mathrm{n}=9)$ aged 8 weeks were obtained from Jackson Laboratories, acclimatized and maintained on a 12-hour light/ dark cycle with provision of Purina Test Diet mouse chow without additives (Purina Mills, LLC) and water ad libitum in pathogen-free microinsulator cages. All procedures were performed according to the humane and customary care and use of experimental animals, and followed a protocol approved by the Internal Animal Care and Use Committee of the Dr. Rath Research Institute.

Treatment groups. The mice were divided into groups as follows: the 9 wild-type mice (Group 1) were harvested at 36 weeks of age as a control group. The $27 \mathrm{ApoE} \mathrm{KO}$ mice were divided into three groups of 9 mice each (Groups 2-4). Group 2 mice were not supplemented with AngII and were harvested at 36 weeks of age. Group 3 mice were implanted with AngIIloaded osmotic pumps (Alzet model 2004, ALZA, Palo Alto, CA, USA) at 32 weeks of age and were harvested 4 weeks later at 36 weeks of age. Group 4 mice were implanted with AngII-loaded osmotic pumps at 36 weeks of age and were harvested at 52 weeks of age. A summary of the treatment groups is shown in Table I.

Angiotensin II osmotic pump installation. Using an anaesthesia machine (VMS Matrx) with a precision isoflurane vaporizer and pressurized oxygen gas, $18 \mathrm{ApoE}(-/-)$ mice were induced before being implanted with osmotic pumps (Alzet Model 2004) in the dorsum subcutaneous pouch. Each pump contained $200 \mu \mathrm{l}$ of $4 \mathrm{mM}$ human AngII, $150 \mathrm{mM} \mathrm{NaCl}$, and $0.01 \mathrm{~N}$ acetic acid (previously sterilized by $0.2 \mu \mathrm{M}$ filtration). Under these conditions, AngII was released at an approximate rate of $27 \mathrm{nmol} / 24 \mathrm{~h}$.

Aortic harvest. Harvests from each group were made at 4 and 16 weeks post-AngII administration. Under isoflurane anesthesia, blood samples were collected through a retrograde left ventricular puncture and stored for biochemical analysis. The viscera and organs were removed, exposing the descending aorta and its branches. Ice cold PBS (500 $\mu \mathrm{l}$ to $1 \mathrm{ml})$ was perfused through the aorta through a left ventricular puncture to flush out the aortic lumen via the abdominal aorta bifurcations. The PBS wash was removed from the renal and hepatic bifurcation area using a syringe without a needle. A dissecting microscope was used to magnify the proximal aorta, the aortic arch, the thoracic aorta and the brachiocephalic, left subclavian and left carotid arteries. The entire aortic tree to the diaphragm was retrieved using micro-forceps and microscissors, and stored individually in formalin after adipose and connective tissue was trimmed in ice cold PBS. The abdominal aorta including the iliac bifurcation was retrieved and stored in the same manner.

Aortic arch and great vessel lesion analysis. Coronal $5-\mu \mathrm{M}$ sections of the formalin-fixed paraffin-embedded aortic tree capturing the entire group of aorta ascendens, braciocephalic (innominate) artery, left carotid, left subclavian, aorta descendens and thoracic aorta were taken from all specimens. Three 5- $\mu \mathrm{M}$ step sections were taken $50 \mu \mathrm{M}$ apart, and then 35 serial sections were taken. Serial sections representative of the brachiocephalic artery lumen throughout each group were selected from 30-35 for Elastin Van Gieson (EVG) staining. Standard EVG staining was performed before the slides were mounted and digital images were captured with a SPOT digital camera at $\mathrm{x} 2$ magnification. Digital planimetry of a defined area of the aortic tree and its major vessels was performed using Scion Image. A semi-circle of the ascending and descending aortic arch was demarcateded by a line drawn from the incision near the aortic root through the opposing descending aorta. An arbitrary maximum length of 150 pixels of the brachiocephalic, left carotid and left subclavian artery areas was included with the aortic arch area measurements. Aortic lesions were graded as per Columbia University Core A definitions based on Reddick et al (7) and Kanters et al $(8,9)$. Type III/Type B aortic dissections, as evidenced by subadventitial thrombus and histological confirmation, were counted per group. Lesion areas were expressed as total lesion area over total lumenal area. Necrotic cores were counted in the measured area of each slide.

Abdominal aneurysm analysis. Wet abdominal aortae with and without aneurysms were taken from formalin and placed in ExCell Plus (American Mastertech Scientific, Inc.) non-formalin fixative overnight. Abdominal aortae were trimmed of adipose tissue under ExCell Plus and moved into the physiological orientation in a $60-\mathrm{mm}$ dish of Excell Plus under a dissecting microscope fitted with a SPOT digital camera. A millimeter ruler was placed in the frame and images were captured at a magnification of $x 1.5$. The maximal diameter of the abdominal aortae and/or their aneurysms was measured against the millimeter ruler in the frame to generate diameter values. 
A

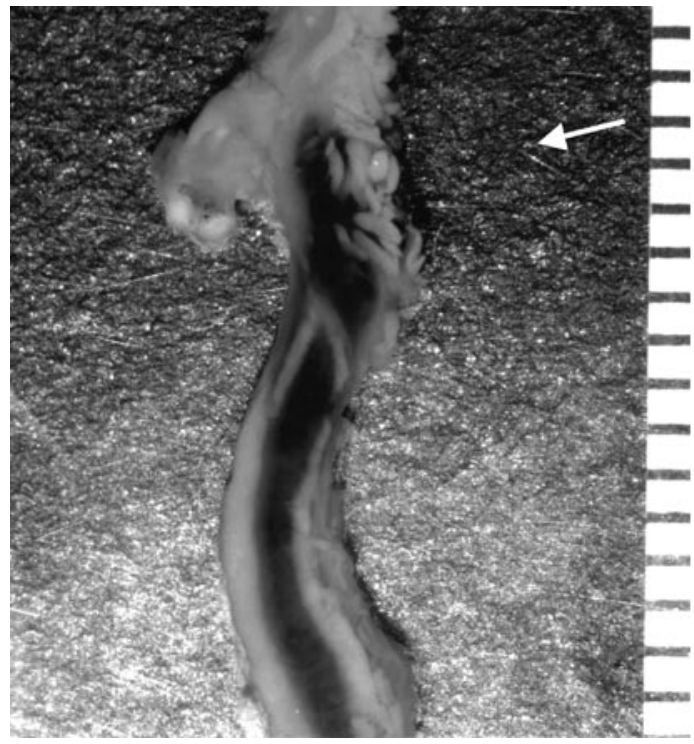

B

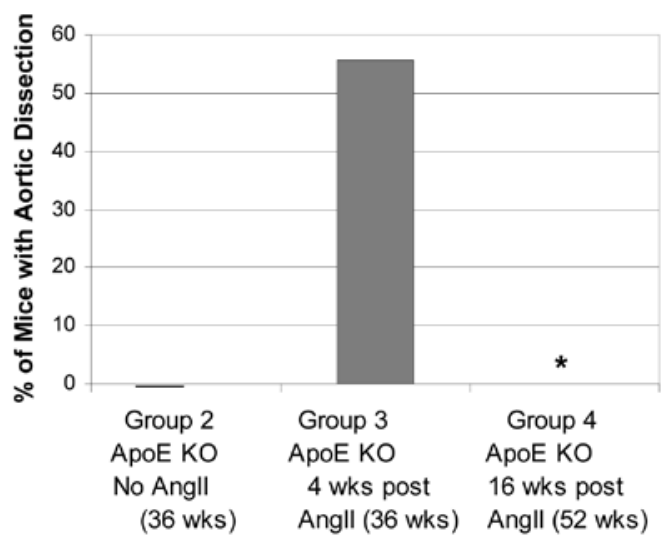

Figure 1. Development of Type B aortic dissection in the groups. (A) Representative aortic dissection in ApoE KO mice after 4 weeks of AngII administration (Group 3). The arrowhead denotes the origin of the break in the lumen (the aortic dissection), which generated a subadventitial flow of blood. The bracket denotes the downward flow of the blood, which sheared the adventitia from the lumen and formed the thrombus within the false lumen. (B) Frequency of aortic dissections in the groups. *Significant difference between Group 3 and Group 4 mice. Wks, weeks.

Statistical analysis. The occurrence of Type B aortic dissections was expressed as percentages and differences between the groups, and analyzed for significance with the $\chi^{2}$ test, using MedCalc (Mariakerke, Belgium). Results for the other parameters were expressed as the means \pm SD of the groups, and differences between the groups were analyzed with the independent samples t-test.

\section{Results}

Formation of Type B aortic dissections by angiotensin II and spontaneous repair after cessation of exogenous angiotensin II. After 4 weeks of AngII administration to ApoE-KO mice (Group 3), dramatic Type B aortic dissections occurred complete with massive sub-adventitial thrombi and false lumens (Fig. 1A). Thoracic dissections, mostly of Type B, occurred with a frequency of $55.6 \%(\mathrm{p}=0.037)$ in the
A

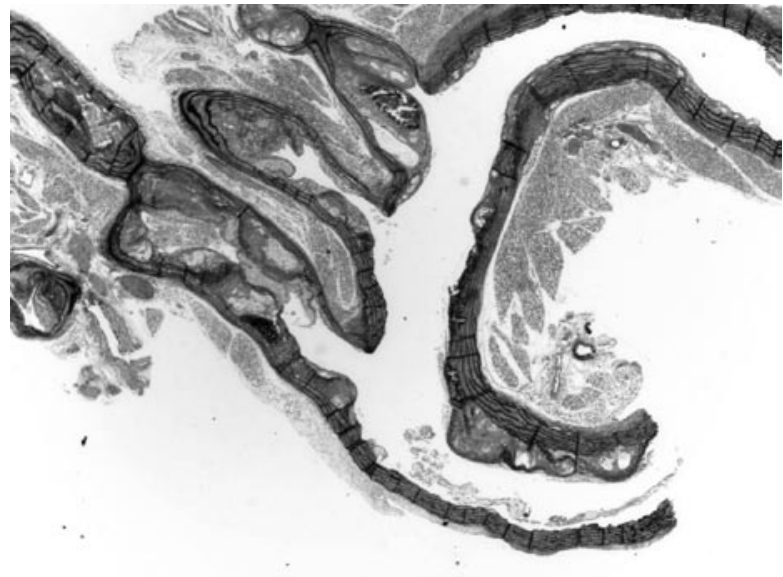

B

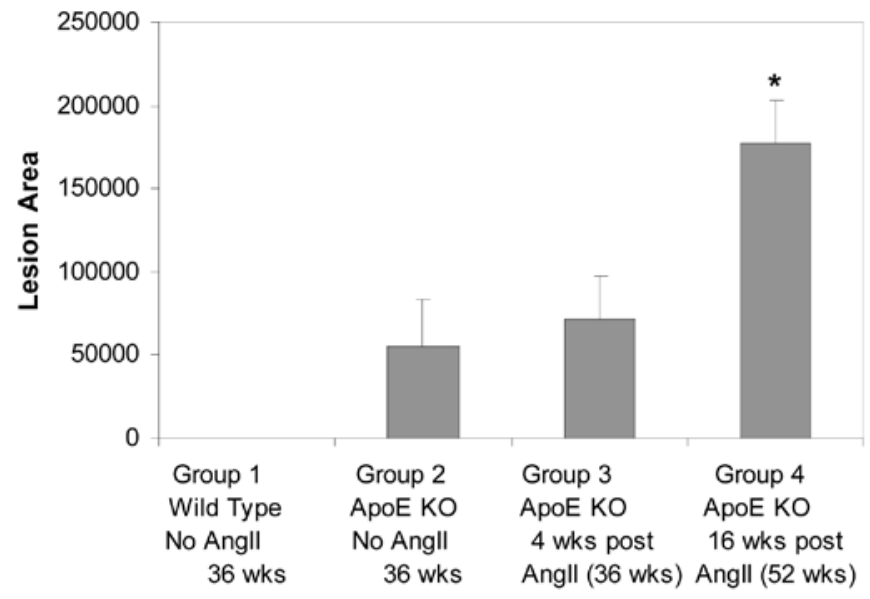

Figure 2. Development of atherosclerotic lesions in arterial trees in the groups. (A) Aortic tree section representative of lesions in ApoE KO mice at 16 weeks post-AngII administration (Group 4). (B) Lesion size differences between the groups. "Significant difference between Group 3 and Group 4 mice. Wks, weeks.

animals administered AngII (Group 3), but did not occur in the age-matched KO control group (Group 2) (Fig. 1B). One Type A/Type II dissection was observed in the AngII group. No aortic dissections nor any traces of false lumens or subadventitial blood or thromboses were found visually or upon histological inspection at 16 weeks post-AngII (Group 4), despite the presence of greatly increased plaque and of abdominal aneurysms. No plaque was found on the distal wall, where the Type B dissections occurred.

Acceleration of aortic arch and great vessel plaque formation after cessation of exogenous angiotensin II. In AngII-treated ApoE KO mice 16 weeks after AngII implantation, the initial injury caused by AngII continued to accelerate lesion size in the major vessels of the aortic arch. Lesion size in the aortas of AngII mice was significantly larger (249\% increase, $\mathrm{p}<0.001)$ at 16 weeks (Group 4) than at 4 weeks (Group 3), suggesting that the deleterious effects of AngII continued even after its levels had returned to normal (Fig. 2). Lesion size in untreated ApoE KO mice was 23\% less than in AngII-treated ApoE KO mice at 4 weeks, but did not reach statistical significance. 


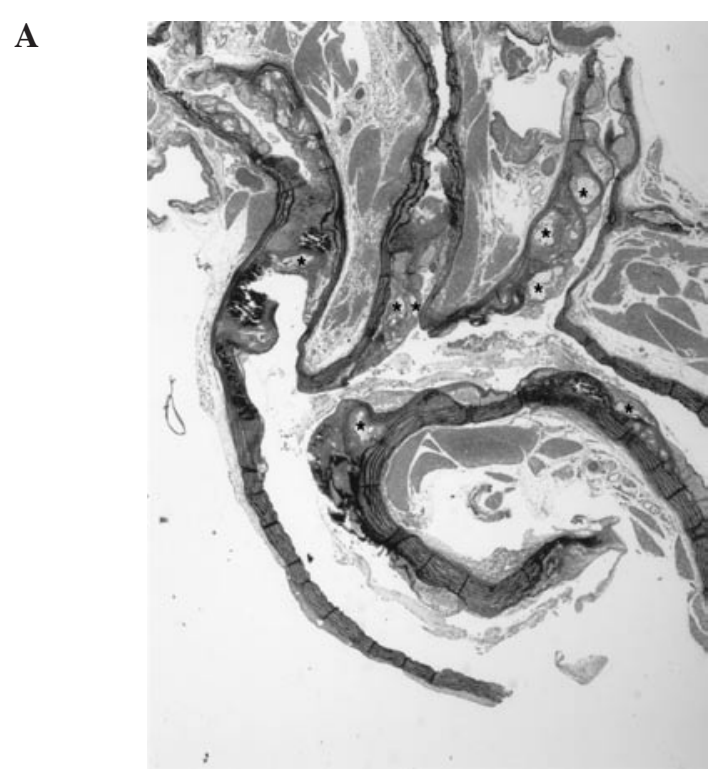

B

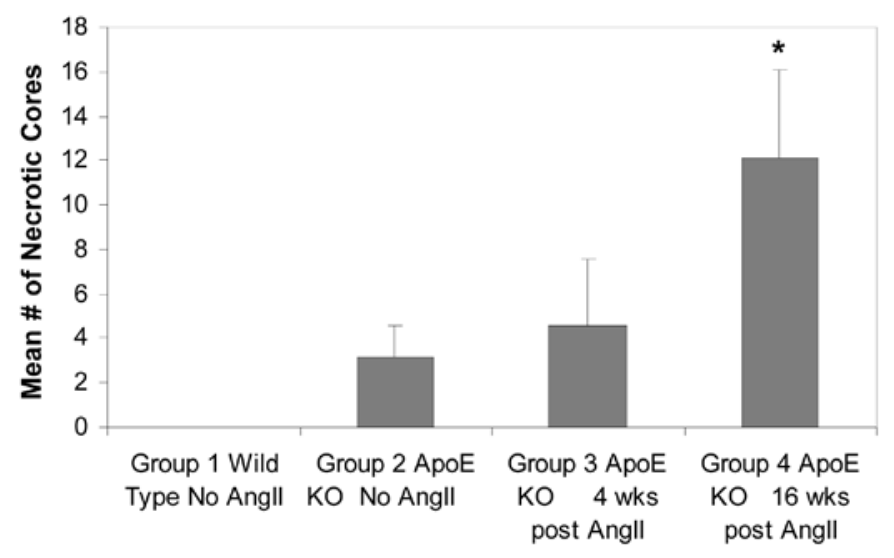

Figure 3. Development of necrotic cores in arterial trees in the groups. (A) Aortic tree section representative of necrotic cores in lesions of AngII-treated ApoE KO mice 16 weeks after AngII osmotic pump implantation (Group 4). (B) Mean number of necrotic cores per arterial tree section in the groups. *Significant difference between Group 3 and Group 4 mice. Wks, weeks.

Wild-type mice did not develop any lesions. After 4 weeks of AngII administration, new lesions continually formed over old lesions. The lesions were more complex at 16 weeks than at 4 weeks. Likewise, the average number of necrotic cores per arterial tree section continued to increase after 4 weeks, resulting in a dramatic difference in the number of necrotic cores between the 4 week post-AngII specimens (Group 3) and 16 week post-AngII specimens (Group 4) (239\% increase, $\mathrm{p}=0.004)$ (Fig. 3). The mean number of necrotic cores in ApoE KO mice treated with AngII (Group 3) was 144\% compared to age-matched ApoE KO untreated mice (Group 2), but this difference did not reach statistical significance $(\mathrm{p}=0.22)$.

Initiation of abdominal aneurysms by angiotensin II and continual dilation after cessation of exogenous angiotensin II. Abdominal aneurysms reached a maximum diameter of $\sim 2.5 \mathrm{~mm}$ at 16 weeks, and did not appear to spontaneously regress at 16 weeks vs. 4 weeks. Abdominal aneurysms formed in response to AngII continued to enlarge after
A

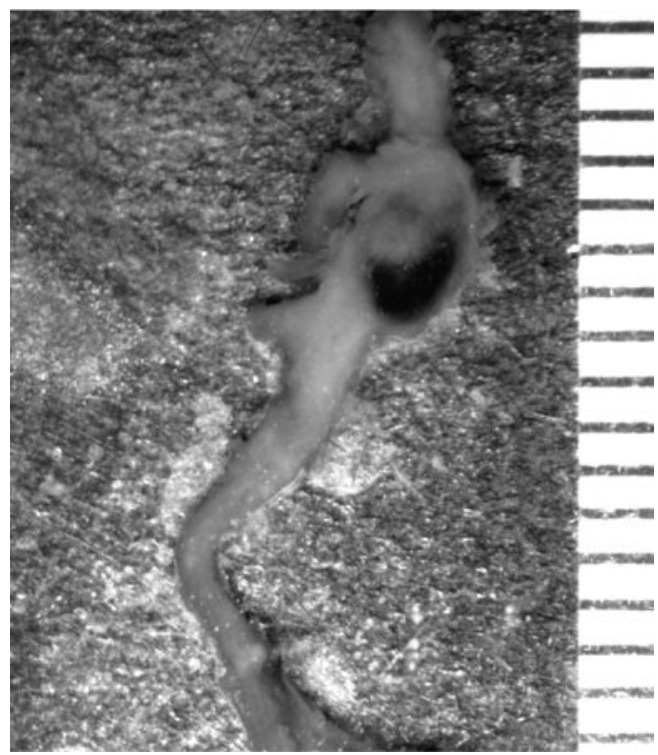

B

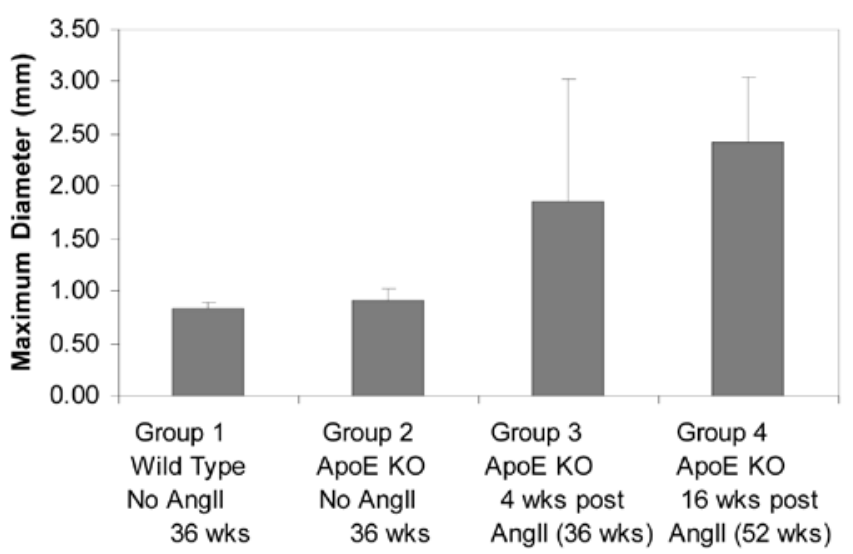

Figure 4. Development of abdominal aneurysms in the groups. (A) Representative photomicrograph of an abdominal aorta in ApoE $\mathrm{KO}$ mice after 4 weeks of AngII administration (Group 3). (B) Mean diameter of the abdominal aortas in the groups. Wks, weeks.

4 weeks, similar to the atherosclerotic lesions of the great vessels at 16 weeks (Group 4) compared to untreated $\mathrm{KO}$ mice (Group 2) $(263 \%$ increase, $\mathrm{p}<0.0001)$. The mean diameter at 16 weeks post-AngII (Group 4) was increased by $130 \%$ compared to that at 4 weeks post-AngII (Group 3), but did not reach statistical significance (Fig. 4B). The mean diameters of the respective groups were as follows: Group 1 (untreated wild-type mice), $0.83 \mathrm{~mm}$; Group 2 (untreated ApoE KO mice), $0.9 \mathrm{~mm}$; Group 3 (ApoE KO mice 4 weeks post-AngII), $1.86 \mathrm{~mm}$; Group 4 (ApoE KO mice 16 weeks post-AngII), $2.42 \mathrm{~mm}$. A photomicrograph of an abdominal aneurysm representative of Group 3 mice is shown in Fig. 4A.

\section{Discussion}

In this study, the AngII-mediated injuries continued to accelerate great vessel atherosclerosis after AngII depletion at 4 weeks. As a result, there was a significant increase in lesion size at 16 weeks compared to that at 4 weeks after the instal- 
lation and administration of AngII in ApoE KO mice. This suggests that excessive AngII initiates a cascade of pathological biochemical events and plaque evolution that does not cease even after its removal. These results corroborate those of Ayabe et al, who found that even transient exposure of ApoE-null mice to AngII primed vessels for increased atherosclerosis 12 weeks later, but not during the infusion (6).

Previously published data suggest that the pathological cascade observed in this study is caused by free radical damage exclusive to the vasopressor AngII and not the vasopressor norepinephrine (10). AngII is known to mediate pathological events in the artery through reactive oxygen species (ROS)-mediated damage, while performing its normal function of vasoconstriction in response to decreased blood volume and mineral salinity. Gavazzi et al demonstrated that while norepinephrine causes $\mathrm{dP} / \mathrm{dT}$ increases by vasoconstriction, it does not generate the vascular lesions that AngII creates (11). Another implicated biochemical mechanism of AngII is the upregulation of MCP-1 and CCR2, which causes the influx of macrophages into the arteries amplifying atherosclerosis long after AngII has washed out (6). Drawing upon the observations of this murine study, it can be extrapolated that the continuation of AngII-mediated atherosclerotic lesion growth after the excessive effects of AngII have been brought under control also occurs in humans. However, whether the initial deleterious effects of excessive AngII on atherosclerosis continue after its reduction to normal levels in humans has yet to be demonstrated. If indeed AngII-initiated atherosclerotic lesions continue to worsen after the normalization of AngII, this may have diagnostic and clinical implications for human hypertension and atherosclerosis.

Human and murine metabolic and cardiovascular systems differ in several important ways. Both murine and human lipoprotein systems carry LDL and HDL, but mice have a higher ratio of HDL making them naturally more resistant to atherosclerosis (12). They also have other genetic differences, such as a functional gulono-lactone-oxidase gene, which makes mice replete with endogenous plasma ascorbic acid. Humans lack the ability to synthesize ascorbate internally and are completely dependant on its nutritional supplementation. Maeda et al demonstrated that the lack of ascorbic acid synthesis in mice causes a breakdown in the arterial wall that does not occur in the presence of ascorbate (13). Rath et al proposed that chronic sub-clinical vitamin $\mathrm{C}$ deficiency is a primary cause of atherosclerotic plaque formation since it leads to the deposition of lipoprotein(a) and fibrinogen/fibrin in the vascular wall. Ascorbic acid is essential for the synthesis and maintenance of collagen and connective tissue, which are critical for blood vessel stability (2). In conjunction with three hydroxylases, it is responsible for post-transcriptional collagen maturation and fibril formation. Immunohistochemical data and electron microscopy show the artery to be a carefully organized arrangement of smooth muscle cells connected to their environment by an autocrine/paracrine-generated matrix of collagen I, collagen III, collagen IV, proteoglycans, laminin and fibronectin. These layers of cells and ECM alternate with layers of elastin, which has mechanical properties of stretch and recoil in contrast to the tensile shear resistance of collagen. Without collagen synthesis, or with abnormal collagen subtype overproduction, the artery becomes fragile or too stiff, respectively (14). Without increasing the tensile strength of the artery in response to hypertension, the human artery may break and hemorrhage, as occurs in the dissecting aorta pathology.

Additionally, ascorbic acid acts as a superoxide radical quencher, and plays a role in mitigating the damage done by ROS generated in the vasculature. It is well documented that NADPH metabolic wastes are responsible for ROS damage in arteries, and that ascorbic acid is an ROS quencher. By virtue of a higher HDL ratio and the endogenous synthesis of ascorbic acid, mice are much less susceptible to the initial-to-advanced atherosclerotic lesions caused by normal metabolism. These are found in humans of all ages, but very rarely in wild type mice $(2,12,15)$. For this reason, laboratories use mice that are modified by knocking out of the ApoE gene, which is critical for a functional reverse cholesterol transport chain. These mice are then fed a diet extremely high in atherogenic saturated fats as well as in cholesterol, or are administered AngII. Other standard murine atherosclerosis models knock out genes at various places in the transport chain of lipoproteins, such as the LDL receptor, thereby causing the abnormal accumulation of LDL in the plasma, the artery or the macrophage.

As previously mentioned, mice generate their own ascorbic acid, while humans must ingest ascorbate in their diet. However, humans have higher uric acid levels in their plasma, which is hypothesized to be an evolutionary compensatory mechanism for missing ascorbate synthesis (13). However, uric acid is not a co-factor of prolyl-hydroxylase, and is known to play a deleterious role by contributing to hypertension (16).

The thrombolytic systems of humans and mice also differ significantly. Murine plasminogen is more resistant to cleavage by tissue plasminogen activator, thereby rendering the mouse physiology less prone to proteolysis by virtue of the decreased liberation of plasmin. Additionally, the less active plasminogen and plasmin of the murine system hypothetically decreases fibrinolysis and thrombus clearance. Empirically, there is sufficient overlap in the reaction of the murine and human thrombolytic systems to be able to infer outcomes in humans based on studies performed in mice. However, the differences between the two are still not fully characterized, and further important differences may be identified in future (17). Fibrinogen is required for the normal function of the wound healing system of mice and humans, and also plays a role in the changes wrought upon the microenvironment of the endothelium and media of the artery. In humans, but not in mice, fibrinogen acts with apo(a) to promote atherosclerosis. Of note, the overexpression of fibrinogen in mice does not promote atherosclerosis unless it is expressed in conjunction with transgenic human apo(a) (17-20).

A notable finding in our study was the frequent presence of aortic dissections at 4 weeks post-AngII administration and the absence of aortic dissections at 16 weeks post-AngII administration. The robust self-repair of aortic dissections in mice has not been observed in humans, for whom mortality continues to increase with time after aortic dissection $(21,22)$. In the treatment of humans, therapeutic interventions for aortic dissection include surgery or stents. While there has been a vast improvement in survival over the last decades, these procedures have not provided dramatic improvements in long- 
term survival. Survival gradually decreases to $5 \%$ at 20 years post-aortic dissection, while generally $27.5 \%$ of patients who have undergone surgery require re-operation after 6 years (21).

This is in stark contrast to the high survivability of aortic dissection in mice observed in this study. Our findings mirror the observation of Saraff et al, that murine aortic dissections are self-repairing albeit with pathological remodeling, followed by atherosclerosis at the same sites (23). This phenomenon warrants further study and evaluation in order to verify the presence of aortic dissection self-repair mechanisms in mice and to determine what the specific genetic and biochemical factors behind them may be. These morphological findings may represent critically important biochemical differences between murine and human atherosclerosis that have been overlooked, and may be phenomena that are not yet characterized in human hypertensive cardiovascular disease. In this study, the presence of ascorbate synthesis in ApoE KO mice and the lack of ascorbate synthesis in humans may explain the differences in the self-repair of aortic dissection in mice vs. humans (2). This self-repair phenomenon may also result from biochemical differences between the proteolytic system and its response to ROS, and the internal production of ascorbate (24).

Biochemically, mice are dramatically dissimilar to humans in their cardiovascular systems. However, murine physiology and biochemistry are similar enough for genetically modified variants to be utilized in models that may provide data that may eventually extend to human treatment. Subtle differences in the anatomical structure and thickness of the intimal and medial layers of the mouse artery versus the human artery, as well as basic differences such as the magnitude of size difference and internal ascorbate synthesis, may be more important considerations than previously realized.

In conclusion, our results suggest that excessive AngII initiates a cascade of pathological biochemical events and plaque evolution that does not cease even after its removal. If the aortic dissection self-repair seen in this mouse study is reproduced in other experimental models and its mechanisms identified, this may have a translational application in the treatment of human aortic dissections, which cause increasing mortality with time despite contemporary surgical intervention. Additionally, the priming of arteries for atherosclerosis by transient exposure to AngII may be an important initiating event in human atherosclerosis. Taken together, these observations may elucidate species differences and similarities of atherosclerosis in response to abnormal AngII signaling, and emphasize the importance of addressing its long-lasting deleterious effects in future research.

\section{Acknowledgements}

This study was funded by the Dr. Rath Health Foundation (Plantation, Florida, USA), a non-profit organization.

\section{References}

1. Schwartz SM, Galis ZS, Rosenfeld ME and Falk E: Plaque rupture in humans and mice. Arterioscler Thromb Vasc Biol 27: 705-713, 2007.

2. Rath M: Solution to the puzzle of human cardiovascular disease: its primary cause is ascorbate deficiency, leading to the deposition of lipoprotein(a) and fibrinogen/fibrin in the vascular wall. J Ortho Med 6: 125-134, 1991.
3. Zadelaar S, Kleemann R, Verschuren L, De Vries-van der Weij J, van der Hoorn J, Princen HM and Kooistra T: Mouse models for atherosclerosis and pharmaceutical modifiers. Arterioscler Thromb Vasc Biol 27: 1706-1721, 2007.

4. Kleemann R, Zadelaar S and Kooistra T: Cytokines and atherosclerosis: a comprehensive review of studies in mice. Cardiovasc Res 79: 360-376, 2008.

5. Whitman SC: A practical approach using mice in atherosclerosis research. Clin Biochem Rev 25: 81-93, 2004.

6. Ayabe N, Babaev VR, Tang Y, Tanizawa T, Fogo AB, Linton MF, Ichikawa Y, Fazio S and Kon V: Transiently heightened angiotensin II has distinct effects on atherosclerosis and aneurysm formation in hyperlipidemic mice. Atherosclerosis 184: 312-321, 2006.

7. Reddick RL, Zhang SH and Maeda N: Atherosclerosis in mice lacking apoE. Evaluation of lesional development and progression. Atheroscler Thromb 14: 141-147, 1994.

8. Kanters E, Pasparakis M, Gijbels MJJ, et al: Inhibition of NF- $\kappa \mathrm{B}$ activation in macrophages increases atherosclerosis in LDL receptor-deficient mice. J Clin Invest 112: 1176-1185, 2003.

9. Kanters E, Gijbels MJJ, van der Made I, Vergouwe MN, Heeringa P, Kraal G, Hofker MH and de Winther MPJ: Hematopoietic NF- $\kappa \mathrm{B} 1$ deficiency results in small atherosclerotic lesions with an inflammatory phenotype. Blood 103: 934-940, 2004.

10. Paravicini TM and Touyz RM: NADPH oxidases, reactive oxygen species, and hypertension: clinical implications and therapeutic possibilities. Diabetes Care 31: S170-S180, 2008.

11. Gavazzi G, Deffert C, Trocme C, Schäppi M, Herrmann FR and Heinz Krause K: NOX1 deficiency protects from aortic dissection in response to angiotensin II. Hypertension 50: 189-196, 2007.

12. Fernandez ML and Volek JS: Guinea pigs: a suitable animal model to study lipoprotein metabolism, atherosclerosis and inflammation. Nutr Metab 3: 17, 2006.

13. Maeda N, Hagihara H, Nakata Y, Hiller S, Wilder J and Reddick R: Aortic wall damage in mice unable to synthesize ascorbic acid. Proc Natl Acad Sci USA 97: 841-846, 2000.

14. Liu X, Wu H, Byrne M, Krane S and Jaenisch R: Type III collagen is crucial for collagen I fibrillogenesis and for normal cardiovascular development. Proc Natl Acad Sci USA 94: 1852-1856, 1997.

15. Nakashima Y, Fujii H, Sumiyoshi S, Wight TN and Sueishi K: Early human atherosclerosis: accumulation of lipid and proteoglycans in intimal thickenings followed by macrophage infiltration. Arterioscler Thromb Vasc Biol 27: 1159-1165, 2007.

16. Sautin YY and Johnson RJ: Uric acid: the oxidant-antioxidant paradox. Nucleosides Nucleotides Nucleic Acids 27: 608-619, 2008.

17. Fay WP, Garg N and Sunkar M: Vascular functions of the plasminogen activation system. Arterioscler Thromb Vasc Biol 27: 1231-1237, 2007.

18. Yano Y, Shimokawa K, Okada Y and Noma A: Immunolocalization of lipoprotein(a) in wounded tissues. J Histochem Cytochem 45: 559-568, 1997.

19. Caplice NM, Panetta C, Peterson TE, Kleppe LS, Mueske CS Kostner GM, Broze GJ Jr and Simari RD: Lipoprotein(a) binds and inactivates tissue factor pathway inhibitor: a novel link between lipoproteins and thrombosis. Blood 98: 2980-2987, 2001.

20. Lou X, Boonmark NW, Horrigan FT, Degen JL and Lawn RM: Fibrinogen deficiency reduces vascular accumulation of apolipoprotein(a) and development of atherosclerosis in apolipoprotein(a) transgenic mice. PNAS 95: 12591-12595, 1998.

21. Fattori R, Bacchi-Reggiani L, Bertaccini P, Napoli G, Fusco F, Longo M, Pierangeli A and Gavelli G: Evolution of aortic dissection after surgical repair. Am J Cardiol 86: 868-872, 2000.

22. Tsai T, Fattori R, Trimarchi S, et al: Long-term survival in patients presenting with type $\mathrm{b}$ acute aortic dissection: insights from the international registry of acute aortic dissection. Circulation 114: 2226-2231, 2006.

23. Saraff K, Babamusta F, Cassis LA and Daugherty A: Aortic dissection precedes formation of aneurysms and atherosclerosis in angiotensin II-infused, apolipoprotein E-deficient mice. Arterioscler Thromb Vasc Biol 23: 1621-1626, 2003.

24. Lind SE, McDonagh JR and Smith CJ: Oxidative inactivation of plasmin and other serine proteases by copper and ascorbate. Blood 82: 1522-1531, 1993. 